\title{
DESENVOLVIMENTO E VALIDAÇÃO DE MÉTODO ANALÍTICO POR CROMATOGRAFIA LÍQUIDA DE ALTA EFICIÊNCIA (CLAE) PARA DETERMINAÇÃO DE ASSOCIAÇÃO DE AMPICILINAS EM PÓ LIOFILIZADO PARA INJEÇÃO
}

\author{
Ana Paola Prata Cione*, Márcio José Liberale e Paulo Marcos da Silva \\ Laboratório de Fármacos, Bioagri Laboratórios, Rod. Rio Claro-Piracicaba, SP 127, km 24, 13412-000 Piracicaba - SP, Brasil
}

Recebido em 15/1/09; aceito em 1/7/09; publicado na web em 8/12/09

\begin{abstract}
DEVELOPMENT AND VALIDATION OF ANALYTICAL METHOD FOR DETERMINATION OF AN ASSOCIATION OF AMPICILLINS IN LYOPHILIZED POWDER FOR INJECTION BY HPLC. An analytical method has been developed and validated for the determination of an association of ampicillins in a lyophilized powder for injection by HPLC. The advantage of chromatographic method other than the microbiological one is that, it is possible to monitor precisely, out-of-specification results in quality control processes and also during stability studies, in which an association of ampicillins is present. The proposed HPLC method was developed by using forced degraded samples, in order to reach a selective analysis of ampicillins when in the presence of their degradation products. It was possible to detect benzatine and through indirect calculation, to determine the ampicillin sodium in the drug sample. The method showed to be selective, accurate, precise, robust and linear (from 45.92 to $36.04 \mu \mathrm{g} \mathrm{mL}{ }^{-1}$ of total ampicillin and from 14.53 to $43.28 \mu \mathrm{g}$ $\mathrm{mL}^{-1}$ of benzatine). The accuracy determined from recovery test, gave results in the range of $99.41 \%$ of total ampicillin to $100.31 \%$ of benzatine. Hence, it can be concluded that the proposed HPLC method is applicable for ampicillins determination.
\end{abstract}

Keywords: ampicillin; HPLC; forced degradation.

\section{INTRODUÇÃO}

Atualmente existem muitas penicilinas diferentes, entretanto, todas são derivadas do ácido 6- aminopenicilânico e possuem uma estrutura básica que consiste num anel tiazolidíco, ligado a um anel beta-lactâmico. O núcleo da penicilina é o principal componente para atividade biológica da substância. Transformações metabólicas ou alterações químicas dessa parte da molécula provocam perda significativa da atividade antibacteriana. A obtenção, por via enzimática, do ácido 6-aminopenicilânico (6-APA) em 1959, por Batchelor et al., ${ }^{1}$ tornou viável a obtenção do núcleo central das penicilinas através de um mecanismo fácil, de elevado rendimento e pouco dispendioso. A partir desses conhecimentos, cientistas iniciaram pesquisas sobre penicilinas semi-sintéticas que apresentassem espectro de ação ampliado em relação à penicilina $\mathrm{G}$ (ou benzilpenicilina). A ampicilina foi a primeira penicilina semi-sintética que mostrou ação contra bacilos gram-negativos, abrindo o campo de penicilinas de amplo espectro. É comercializada na forma anidra, tri-hidratada e sódica, bem como nas formas de depósito, isto é, ligada à benzatina. A Figura 1 apresenta a estrutura química da molécula de ampicilina.<smiles>CC1(C)S[C@@H]2[C@H](NC(=O)[C@@H](N)c3ccccc3)C(=O)N2[C@H]1C(=O)O</smiles>

Figura 1. Estrutura química da ampicilina

A literatura indica uma variedade de métodos para determinação de ampicilina ou "ampicilina total". Estes métodos incluem determinações microbiológicas, o método farmacopêico iodométrico, ${ }^{2}$ por cromatografia de troca iônica e eletroforese. ${ }^{3}$ Há também uma vasta

*e-mail: anapaola@bioagri.com.br literatura sobre a determinação por CLAE de ampicilina em fluidos, como plasma ${ }^{4}$ e urina ${ }^{5}$ e, nesses casos, há menção de uso de detectores mais sensíveis e seletivos, como espectrômetros de massas.

Ainda, podem ser encontrados alguns métodos para a determinação de ampicilinas em associação, por cromatografia líquida, ${ }^{6}$ espectrofotometria, ${ }^{7}$ porém, a maioria deles envolve o uso de fases móveis não convencionais, como no caso da cromatografia líquida micelar $^{8}$ ou até mesmo uso de instrumentação específica como um FIA acoplado a detector de luminescência. ${ }^{9}$

O uso de métodos indicadores de estabilidade (MIE), ${ }^{10}$ seletivos aos princípios ativos e seus produtos de degradação, tem sido altamente recomendado pela Agência Nacional de Vigilância Sanitária (ANVISA) ${ }^{10}$ para acompanhamento de resultados provenientes de estudos de estabilidade de medicamentos. ${ }^{11}$ A estabilidade de penicilinas tem sido estudada ${ }^{9,12}$ com o objetivo de se estender o tempo de validade das soluções extemporâneas, por exemplo, e o uso de métodos analíticos confiáveis, capazes de determinar os possíveis produtos de degradação é desejável nesses casos.

O principal objetivo desse trabalho foi propor um método cromatográfico e indicador de estabilidade, para determinação de ampicilina total e benzatina por cromatografia líquida com detecção na região do ultravioleta, utilizando-se detector convencional de arranjo de diodos (DAD) e amostras submetidas a condições de estresse (degradação forçada). Nos casos em que duas formas de penicilina estejam presentes em um mesmo medicamento, é possível a determinação quantitativa de ambas com resolução adequada entre os picos.

Uma das vantagens desse método é que torna possível a pesquisa de erros ${ }^{13}$ durante o processo de produção do medicamento (controle de qualidade), bem como durante estudos de estabilidade, ${ }^{11}$ no qual estejam presentes os dois princípios ativos. Deve-se salientar que a determinação microbiológica de ampicilinas é limitada neste aspecto. Além disso, não são encontradas, até o momento, na literatura menções a respeito de métodos cromatográficos indicadores de estabilidade para ampicilinas.

Após o desenvolvimento e otimização do método, o mesmo foi devidamente validado, ${ }^{10}$ por ensaios de especificidade/seletividade, 
linearidade, exatidão, precisão, limite de detecção (LD), limite de quantificação (LQ) e robustez.

\section{PARTE EXPERIMENTAL}

\section{Soluções}

Preparação da solução de fase móvel: (A) Tampão Fosfato de Potássio dibásico pH 3,7 e (B) Metanol.

Preparação da solução diluente: 1000 mL com água ultra-pura e ajustou-se o pH para 3,0 com ácido fosfórico 85\% (v:v).

\section{Preparação das amostras}

Pesaram-se cerca de $30 \mathrm{mg}$ de uma amostra comercial (pó liofilizado para reconstituição e injeção) e transferiu-se para balão volumétrico de $25 \mathrm{~mL}$; completou-se o volume com solução diluente. Retirou-se uma alíquota de $1 \mathrm{~mL}$ desta solução e transferiu-se para um balão volumétrico de $10 \mathrm{~mL}$, completou-se o volume com a solução diluente. Agitou-se em vórtex por $30 \mathrm{~s}$ e filtrou-se através de membrana de fluoreto de polivinilideno (PVDF) $0,45 \mu \mathrm{m}$.

\section{Preparação dos padrões}

Pesaram-se cerca de $25 \mathrm{mg}$ de padrão de Ampicilina Benzatina e $5 \mathrm{mg}$ de padrão de Ampicilina Sódica (Bioquim Liofilizaciones, S.A.) e transferiram-se as massas para um balão volumétrico de 25 $\mathrm{mL}$, diluiu-se em solução diluente. Retirou-se uma alíquota de 1 $\mathrm{mL}$ e transferiu-se para um balão volumétrico de $10 \mathrm{~mL}$, diluiu-se com solução diluente. Desta forma a concentração da solução será, em relação à Ampicilina Benzatina de $69,70 \mu \mathrm{g} \mathrm{mL} \mathrm{m}^{-1}$ (corrigida pela pureza de 69,7\%) e em relação à Ampicilina Sódica de 19,12 $\mu \mathrm{g}$ $\mathrm{mL}^{-1}$ (corrigida pela pureza de 95,6\%), de Benzatina: $28, \mu \mathrm{g} \mathrm{mL} \mathrm{m}^{-1} \mathrm{e}$ de Ampicilina Total: 87,64 $\mu \mathrm{g} \mathrm{mL}^{-1}$.

\section{Equipamentos e condições cromatográficas}

Utilizou-se um cromatógrafo líquido da marca Agilent, modelo HP 1100 equipado com Detector UV-DAD. A coluna empregada foi a X-Terra RP 8 com dimensões de 4,6 x $250 \mathrm{~mm}$, diâmetro de partícula de $5 \mu \mathrm{m}$ e temperatura do forno em $35^{\circ} \mathrm{C}$. A fase móvel consistiu de tampão fosfato de potássio dibásico pH 3,7 e metanol nas proporções $80: 20 \mathrm{v} / \mathrm{v}$ em um fluxo de $1,2 \mathrm{~mL} \mathrm{~min}$ ${ }^{1}$. O volume de injeção foi de $5 \mu \mathrm{L}$. O comprimento de onda de detecção foi de $215 \mathrm{~nm}$ (fenda $4 \mathrm{~nm}$ ) e o comprimento de onda de referência foi de $360 \mathrm{~nm}$ (fenda $4 \mathrm{~nm}$ ). Nestas condições os tempos de retenção da Benzatina e da Ampicilina Total foram de aproximadamente 3,5 e 4 min, respectivamente. Obs.: O pico cromatográfico da Ampicilina Total refere-se à molécula de ampicilina proveniente da Ampicilina Sódica mais a ampicilina proveniente da Ampicilina Benzatina.

\section{Especificidade/seletividade}

A Tabela 1 apresenta a forma de preparo das amostras para ensaio de especificidade/seletividade. A especificidade/seletividade foi avaliada após estresse da amostra em condições de hidrólise ácida, hidrólise básica, oxidação por peróxido de hidrogênio, exposição à luz e calor, por um período de $120 \mathrm{~h}$.

Após o desenvolvimento do método, o mesmo foi devidamente validado para os ensaios de linearidade, exatidão, precisão e robustez. ${ }^{14-17}$

\section{Linearidade}

A linearidade foi testada entre 50 e $150 \%$ da concentração nominal de Ampicilina Total e de Benzatina na amostra, tendo-se construído uma curva entre 45,92 e 136,04 $\mu \mathrm{g} \mathrm{mL} \mathrm{m}^{-1}$ para Ampicilina Total e 14,53 e 43,28 $\mu \mathrm{g} \mathrm{mL}^{-1}$ para Benzatina. As soluções de padrão foram injetadas em triplicata em cada um dos cinco níveis. O limite de detecção (LD) e o limite de quantificação (LQ) foram determinados a partir das curvas de linearidade de Ampicilina Total e Benzatina, como 3 x s/ic e $10 \mathrm{x}$ s/ic, respectivamente, onde s é desvio padrão entre os valores de intercepto com o eixo Y das três curvas e ic e a inclinação da curva. ${ }^{6}$

\section{Exatidão}

As amostras para o ensaio de exatidão foram preparadas pela adição de Ampicilina Benzatina e Ampicilina Total ao veículo, neste caso água, em três níveis, 50, 100 e 150\% da concentração nominal de Ampicilina Total e Benzatina na amostra.

\section{Precisão}

A preparação das amostras utilizadas para determinação da precisão em termos de repetitividade e precisão intermediária seguiu o procedimento descrito no item "preparação das amostras" e realizado para seis réplicas. A precisão intermediária consistiu da preparação de novas amostras, por um analista diferente daquele que preparou as amostras da repetitividade e analisou-se dois dias após o ensaio de repetitividade.

A Equação 1 expressa o cálculo para obtenção do teor de Ampicilina Benzatina e a Equação 2, o cálculo para o teor de Ampicilina Total:

$$
\begin{aligned}
& \mathrm{C}(\mathrm{mg} / \mathrm{ampola})=\mathrm{Rc} \times 250 / \mathrm{m} \times 589 / 240 \times \mathrm{PM} / 1000 \\
& \mathrm{C}(\mathrm{mg} / \mathrm{ampola})=\mathrm{Rc} \times 250 / \mathrm{m} \times \mathrm{PM} / 1000
\end{aligned}
$$

onde: $\mathrm{C}(\mathrm{mg} / \mathrm{ampola})$ = concentração calculada de Ampicilina Bbenzatina; $\mathrm{R}_{\mathrm{c}}=$ resultado obtido no cromatograma (pico cromatográfico Benzatina) $\left(\mu \mathrm{g} \mathrm{mL} \mathrm{m}^{-1}\right) ; 250=$ volume de diluição $(\mathrm{mL}) ; \mathrm{m}=$ massa da tomada de ensaio (g); 589 = massa molar da Ampicilina Benzatina (g); 240 = massa molar da Benzatina (g); PM = peso médio da ampola $(\sim 0,3 \mathrm{~g})$.

\begin{tabular}{|c|c|c|c|c|}
\hline Condição de degradação & $\begin{array}{c}\text { Massa Ampicilina } \\
\text { Benzatina (g) }\end{array}$ & $\begin{array}{l}\text { Massa Ampicilina } \\
\text { Sódica }(\mathrm{g})\end{array}$ & $\begin{array}{c}\text { Solução Reagente utilizada } \\
\text { na degradação }\end{array}$ & $\begin{array}{l}\text { Solução Reagente utilizada } \\
\text { ao final da degradação }\end{array}$ \\
\hline Hidrólise ácida ${ }^{(a)}$ & 25 & 4 & $\mathrm{HCl} 0,1 \mathrm{~mol} \mathrm{~L}^{-1}$ & $\mathrm{NaOH} 0,1 \mathrm{~mol} \mathrm{~L}^{-1}$ \\
\hline Hidrólise básica ${ }^{(a)}$ & 25 & 4 & $\mathrm{NaOH} 0,1 \mathrm{~mol} \mathrm{~L}^{-1}$ & $\mathrm{HCl} 0,1 \mathrm{~mol} \mathrm{~L}^{-1}$ \\
\hline Oxidação $^{(a)}$ & 25 & 4 & $\mathrm{H}_{2} \mathrm{O}_{2} 3 \%(\mathrm{v} / \mathrm{v})$ & Água \\
\hline $\mathrm{Luz}^{(\mathrm{b})}$ & 25 & 4 & Água & Água \\
\hline Calor $^{(\mathrm{c})}$ & 25 & 4 & Água & Água \\
\hline
\end{tabular}

Tabela 1. Preparo de amostras para ensaio de degradação forçada (seletividade)

(a) Amostra mantida em estufa em $60{ }^{\circ} \mathrm{C}$; (b) exposição à lâmpada de arco de xenônio $\left(265 \mathrm{~W} \mathrm{~m}^{-2}\right)$; ${ }^{(\mathrm{c})}$ amostra mantida em estufa em $60{ }^{\circ} \mathrm{C}$. 
A quantidade de Ampicilina Sódica (Equação 3 - mg/ampola) é obtida pela diferença entre o teor de Ampicilina Total (Equação 2) e o teor de Ampicilina Benzatina (Equação 1):

ampicilina sódica $=$ ampicilina total - ampicilina benzatina

\section{RESULTADOS E DISCUSSÃO}

Nos itens a seguir, estão apresentados os resultados dos ensaios de especificidade, linearidade, LD e LQ, precisão, exatidão e robustez do método.

\section{Especificidade}

Nas Tabelas 2 e 3 estão apresentados os resultados referentes ao testes de especificidade realizados pela adição de padrão ao veículo da amostra (neste caso, água - Figura 2) após exposição da amostra a cada uma das seis condições de estresse (hidrólise ácida e básica, oxidação, luz e calor). Estão apresentadas também a concentração

Tabela 2. Resultados de recuperação do ensaio especificidade - Benzatina

\begin{tabular}{lccccc}
\hline Teste & $\begin{array}{c}\text { Tomada de } \\
\text { ensaio }\end{array}$ & $\begin{array}{c}\text { Conc. } \\
\text { Adic. }\end{array}$ & $\begin{array}{c}\text { Conc. } \\
\text { Recup. }\end{array}$ & Recup. & $\begin{array}{c}\text { Recup. } \\
\text { Média }\end{array}$ \\
\hline Degradação & $(\mathrm{mg})$ & $\mu \mathrm{g} / \mathrm{mL}$ & $\mu \mathrm{g} / \mathrm{mL}$ & $(\%)$ & $(\%)$ \\
Controle-1 & 5,90 & 25,53 & 25,18 & 98,64 & 98,93 \\
Controle-2 & 5,88 & 26,67 & 26,46 & 99,23 & \\
$\mathrm{HCl}-1$ & 25,40 & 28,82 & 28,85 & 77,70 & 77,93 \\
$\mathrm{HCl}-2$ & 25,48 & 28,91 & 22,60 & 78,15 & \\
$\mathrm{NaOH}-1$ & 25,69 & 29,15 & 25,75 & 89,15 & 92,28 \\
$\mathrm{NaOH}-2$ & 25,62 & 29,07 & 27,68 & 95,40 & \\
$\mathrm{H}_{2} \mathrm{O}_{2}-1$ & 25,67 & 29,12 & 4,05 & 13,92 & 12,31 \\
$\mathrm{H}_{2} \mathrm{O}_{2}-2$ & 25,62 & 29,07 & 3,11 & 10,71 & \\
Luz- 1 & 25,07 & 28,44 & 20,46 & 71,93 & 72,18 \\
Luz -2 & 25,11 & 28,49 & 20,64 & 72,43 & \\
Calor-1 & 25,78 & 29,25 & 0,80 & 2,72 & 3,56 \\
Calor-2 & 25,59 & 29,03 & 1,28 & 4,40 & \\
\hline
\end{tabular}

Tabela 3. Resultados de recuperação do ensaio especificidade - Ampicilina Total

\begin{tabular}{lccccc}
\hline Teste & $\begin{array}{c}\text { Tomada de } \\
\text { ensaio }\end{array}$ & $\begin{array}{c}\text { Conc. } \\
\text { Adic. }\end{array}$ & $\begin{array}{c}\text { Conc. } \\
\text { Recup. }\end{array}$ & Recup. & $\begin{array}{c}\text { Recup. } \\
\text { Média }\end{array}$ \\
\hline Degradação & $(\mathrm{mg})$ & $\mu \mathrm{g} / \mathrm{mL}$ & $\mu \mathrm{g} / \mathrm{mL}$ & $(\%)$ & $(\%)$ \\
Controle-1 & 22,50 & 85,29 & 85,77 & 100,57 & 100,40 \\
Controle-2 & 23,51 & 88,03 & 88,23 & 100,22 & \\
$\mathrm{HCl}-1$ & 6,10 & 94,14 & 7,07 & 7,51 & 6,55 \\
$\mathrm{HCl}-2$ & 6,12 & 94,44 & 5,28 & 5,59 & \\
$\mathrm{NaOH}-1$ & 5,12 & 00,00 & 0,00 & 00,00 & 00,00 \\
$\mathrm{NaOH}-2$ & 5,14 & 00,00 & 0,00 & 00,00 & \\
$\mathrm{H}_{2} \mathrm{O}_{2}-1$ & 5,67 & 00,00 & 0,00 & 00,00 & 00,00 \\
$\mathrm{H}_{2} \mathrm{O}_{2}-2$ & 5,69 & 00,00 & 0,00 & 00,00 & \\
Luz- 1 & 5,02 & 89,09 & 70,62 & 79,26 & 79,88 \\
Luz -2 & 5,09 & 89,47 & 72,02 & 80,50 & \\
Calor-1 & 5,72 & 00,00 & 0,00 & 00,00 & 00,00 \\
Calor-2 & 5,68 & 00,00 & 0,00 & 00,00 & \\
\hline
\end{tabular}

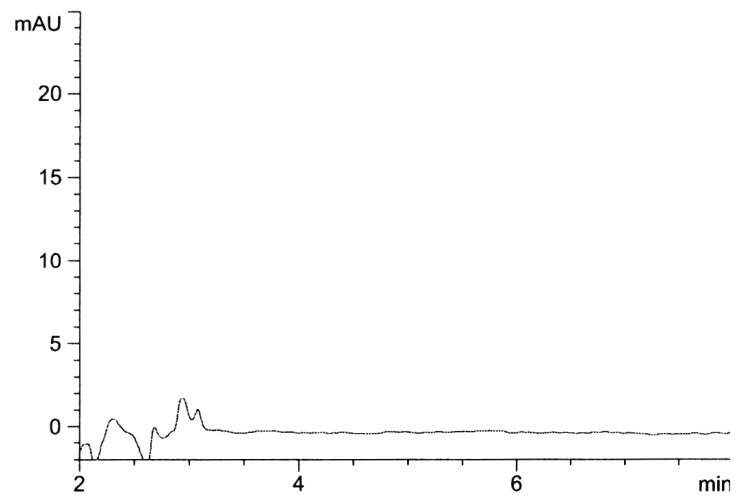

Figura 2. Cromatograma representativo do branco

teórica esperada, a concentração obtida e os resultados de recuperação (individuais e a média) em porcentagem, para a Benzatina e para ampicilina total, respectivamente.

O ensaio que gerou degradação substancial da molécula de Benzatina foi aquele no qual a amostra foi exposta ao calor, tendo a concentração reduzida a aproximadamente $3 \%$ da concentração inicial (Figura 1S, material suplementar). Na amostra em peróxido de hidrogênio, observou-se também uma degradação expressiva, com recuperação de aproximadamente $12 \%$ da concentração inicial (Figura 2S, material suplementar). O pico cromatográfico da Benzatina que aparece em aproximadamente 3,5 min apresentou-se puro, comprovando a ausência de interferentes neste tempo de retenção. Para as demais condições como exposição à luz, hidrólise ácida, hidrólise básica, a porcentagem de recuperação variou entre 72 e $92 \%$. A amostra controle (armazenada em vidro âmbar e mantida em bancada) apresentou $98 \%$ de recuperação.

Nesses mesmos cromatogramas, observa-se que o pico cromatográfico da Ampicilina Total que aparecia em aproximadamente 4 min, desapareceu, indicando $100 \%$ de degradação deste princípio ativo nestas condições (meio peróxido de hidrogênio e calor - 60 ${ }^{\circ} \mathrm{C}$ ). Observou-se igualmente degradação total na amostra em meio básico. As amostras expostas à luz apresentaram cerca de $20 \%$ de degradação e em meio ácido, $83 \%$ de degradação.

As amostras degradadas foram utilizadas durante o desenvolvimento do método cromatográfico, de forma a se atingir uma condição analítica (coluna, fase móvel, comprimentos de onda etc.) apropriada, ou seja, que apresentassem resolução maior que 2,0 entre os picos cromatográficos da Benzatina e da Ampicilina Total, bem como picos simétricos e principalmente "puros", ou seja, não coeluindo com os produtos de degradação formados. O fator de pureza foi fornecido pelo software do cromatógrafo, utilizando um threshold de 990.

\section{Linearidade}

As curvas de linearidade foram testadas no intervalo de 50 a $150 \%$ da concentração nominal - $29 \mu \mathrm{g} \mathrm{mL}^{-1}$ para Benzatina e 92 $\mu \mathrm{g} \mathrm{mL^{-1 }}$ para Ampicilina Total. O coeficiente de regressão linear, r, obtido para ambas as curvas foi maior que 0,999 e o fator de resposta apresentou-se constante em todos os níveis de concentração testados.

A análise da variância (ANOVA) retornou valores de $\mathrm{F}_{\text {calculado }}<$ $\mathrm{F}_{\text {tabelado }}$. Para $\mathrm{p}=2 ; \mathrm{m}=5 ; \mathrm{n}=3$ o $\mathrm{F}_{(\mathrm{m}-\mathrm{p}),(\mathrm{n}-\mathrm{m})}=\mathrm{F}_{(3,10)}=3,71 . \mathrm{O}$ valor de $\mathrm{F}_{\text {calculado }}$ para a curva de linearidade da Ampicilina Total foi de 3,49 e da Benzatina foi de 3,19. Com base nestes resultados, a hipótese nula não pode ser rejeitada, indicando que o ensaio de linearidade pode ser considerado satisfatório. 
Os limites de detecção e quantificação foram determinados como

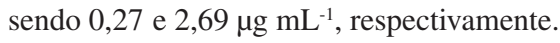

\section{Exatidão}

A Tabela 4 apresenta os resultados da exatidão da Ampicilina total e da Benzatina em três níveis, em relação à concentração nominal na amostra.

Os valores obtidos da exatidão em cada nível apresentaram-se próximos de $100 \%$ sendo que a média geral foi de $100,31 \%$ com um desvio de $0,20 \%$, indicando que o método apresenta recuperação dentro das especificações estabelecidas. ${ }^{10}$

Os resultados obtidos no ensaio de recuperação da Ampicilina Total são proporcionais ao da Benzatina indicando, nesse caso, que a forma de preparo da amostra é eficiente para extração da Benzatina, mas também da ampicilina ligada ao sódio.

Tabela 4. Resultados do ensaio de exatidão - Benzatina e Ampicilina total

\begin{tabular}{|c|c|c|c|c|}
\hline \multirow[t]{2}{*}{ Nível } & \multicolumn{2}{|c|}{ Benzatina } & \multicolumn{2}{|c|}{ Ampicilina total } \\
\hline & $\begin{array}{c}\text { Recup. } \\
(\%)\end{array}$ & $\begin{array}{c}\text { Recup. } \\
\text { Média }(\%)\end{array}$ & $\begin{array}{c}\text { Recup. } \\
(\%)\end{array}$ & $\begin{array}{c}\text { Recup. } \\
\text { Média (\%) }\end{array}$ \\
\hline $50 \%$ Rep. 1 & 99,58 & 100,48 & 99,54 & 99,29 \\
\hline $50 \%$ Rep. 2 & 101,10 & & 99,54 & \\
\hline $50 \%$ Rep. 3 & 100,77 & & 98,81 & \\
\hline $100 \%$ Rep. 1 & 100,11 & 100,09 & 98,96 & 99,54 \\
\hline 100\% Rep. 2 & 99,85 & & 99,96 & \\
\hline $100 \%$ Rep. 3 & 100,30 & & 99,71 & \\
\hline $150 \%$ Rep. 1 & 100,40 & 100,36 & 99,73 & 99,40 \\
\hline $150 \%$ Rep. 2 & 100,73 & & 99,41 & \\
\hline \multirow[t]{4}{*}{$150 \%$ Rep. 3} & 99,96 & & 99,06 & \\
\hline & Média & 100,31 & & 99,41 \\
\hline & DP & 0,20 & & 0,12 \\
\hline & DPR & 0,20 & & 0,13 \\
\hline
\end{tabular}

\section{Precisão}

Os resultados obtidos no ensaio de precisão em termos de repetitividade e precisão intermediária para Benzatina e Ampicilina Total estão apresentados na Tabela 5. Estão expressos em mg/ampola, conforme especificação do produto comercial. As amostras para este ensaio foram preparadas conforme item "preparação das amostras", em seis réplicas.

Os resultados indicam valores de repetitividade e precisão intermediária com valor médio de 173,09 mg/ampola e DPR entre os resultados da repetitividade e da precisão intermediária de $0,52 \%$. Foi encontrado um valor médio de $222,58 \mathrm{mg} /$ ampola de ampicilina total, com DPR geral de 1,03\%, comprovando a precisão do método, para ambos os picos. A Figura 3 apresenta um cromatograma representativo do ensaio de precisão.

\section{Robustez}

Com o objetivo de testar a robustez do método foram testadas duas variações em relação ao método original: fluxo da fase móvel $\left( \pm 0,1 \mathrm{~mL} \mathrm{~min}^{-1}\right)$ e proporção de metanol na fase móvel $( \pm 0,5 \% \mathrm{v} / \mathrm{v})$. A comparação entre os desvios do método testado com o obtido no ensaio de precisão no método original forneceu desvios menores que $2 \%$ (Tabela 1S, material suplementar). Desta forma, o método foi considerado robusto frente às modificações realizadas.
Tabela 5. Resultados do ensaio de precisão - Benzatina e Ampicilina total

\begin{tabular}{lcccc}
\hline & \multicolumn{2}{c}{ Benzatina } & \multicolumn{2}{c}{ Ampicilina Total } \\
Amostras & $\begin{array}{c}\text { Repetitividade } \\
\text { (mg/amp. })\end{array}$ & $\begin{array}{c}\text { Precisão } \\
\text { Intermediária } \\
\text { (mg/amp. })\end{array}$ & $\begin{array}{c}\text { Repetitividade } \\
\text { (mg/amp. })\end{array}$ & $\begin{array}{c}\text { Precisão } \\
\text { Intermediária } \\
\text { (mg/amp. })\end{array}$ \\
\hline Amostra 1 & 173,14 & 173,23 & 220,71 & 222,99 \\
Amostra 2 & 173,94 & 172,50 & 220,82 & 222,22 \\
Amostra 3 & 174,05 & 172,66 & 220,95 & 226,80 \\
Amostra 4 & 173,76 & 172,88 & 219,68 & 226,60 \\
Amostra 5 & 173,90 & 170,88 & 221,48 & 223,90 \\
Amostra 6 & 173,96 & 173,36 & 221,10 & 223,68 \\
Média & 173,79 & 172,59 & 220,79 & 224,37 \\
DP & 0,33 & 0,89 & 0,61 & 1,90 \\
DPR & 0,19 & 0,52 & 0,28 & 0,85 \\
Média Geral & 173,19 & & 222,58 & \\
DP Geral & 0,90 & & 2,30 & \\
DPR Geral & 0,52 & & 1,03 & \\
\hline Peso mé & & & & \\
\hline
\end{tabular}

Peso médio/ampola $=0,300 \mathrm{~g}$

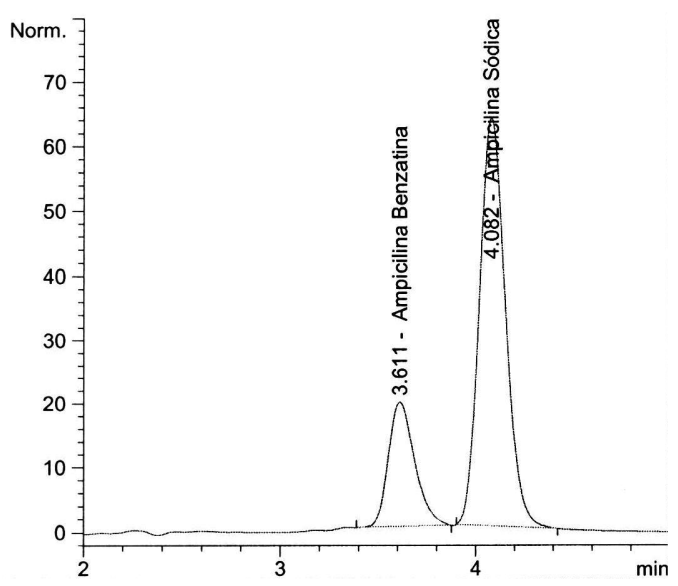

Figura 3. Cromatograma representativo do ensaio de precisão

\section{CONCLUSÕES}

A análise dos resultados obtidos a partir do método desenvolvido forneceu dados que comprovam que o mesmo é válido, uma vez que todos os ensaios (linearidade, LD, LQ, precisão, exatidão, robustez) atenderam às exigências analíticas. Esse método pode ser aplicado aos processos de controle de qualidade e estudos de estabilidade de medicamentos contendo Ampicilina Benzatina e Ampicilina Sódica - em medicamentos nos quais a associação destes princípios ativos esteja presente, uma vez que os ensaios de seletividade foram realizados na presença de produtos de degradação. Além disso, o método proposto por CLAE, mais rápido e eficiente, aparece como uma alternativa válida para a substituição ou complementação do método farmacopêico microbiológico.

\section{MATERIAL SUPLEMENTAR}

Está disponível em http://quimicanova.sbq.org.br, na forma de arquivo .PDF, com acesso livre. 


\section{REFERÊNCIAS}

1. Batchelor, F. R.; Doyle, F. P.; Nayler, J. H.; Rolinson, G. N.; Nature 1959, 183, 257.

2. The United States Pharmacopeia/The National Formulary; 31 ed, Pharmacopoeial Convention: Rockville, 2008.

3. Tsuji, K.; Robertson, J. H.; J. Pharm. Sci. 1975, 64, 1542.

4. Rosseel, M. T.; Bogaert, M. G.; Valcke, Y. J.; Chromatographia 1989, $27,243$.

5. Haginaka, J.; Wakai, J.; J. Pharm. Pharmacol. 1987, 39, 5.

6. Kumar, V.; Bhutani, H.; Singh, S.; J. Pharm. Biomed. Anal. 2007, 43, 769; Hsu, M-C.; Hsu P-W.; Antimicrob. Agents Chemoter. 1992, 36, 1276.

7. Morelli, B.; J. Pharm. Sci. 1988, 77, 1042; Akkani, A. O.; Ayim, J. S. K., J. Pharm. Biomed. Anal. 1992, 10, 43.

8. Srisom, P.; Liawruangrath, B.; Liawruangrath S.; Chromatographia 2007, 65, 687.

9. Li, Y.; Tang, Y.; Yao, H. Y.; Fu, J.; Luminescence 2003, 18, 313.
10. Agência Nacional de Vigilância Sanitária (ANVISA); Guia para Validação de Métodos Analíticos e Bioanalíticos, Resolução RE n 899 de 29/05/2003.

11. Agência Nacional de Vigilância Sanitária (ANVISA); Guia para a Realização de Estudos de Estabilidade, Resolução RE n 01, de 29/05/2005.

12. Allwood, M. C.; Brown, P. W.; Int. J. Pharm. 1993, 9, 219.

13. Guindance for Industry - Investigating Out-of-Specification (OOS)- Test Results for Pharmaceutical Production; Food and Drug Administration (FDA), Center for Drug Evaluation (CDER), Rockville, 2006.

14. Ruela, A. L. M.; Araújo, M. B.; Pereira, G. R.; Quim. Nova 2009, 32, 165.

15. Fregonezi-Nery, M. M.; Baracat, M. M.; Casagrande, B.; Machado, H. T.; Miglioranza, B.; Gianotto, E. A. S.; Quim. Nova 2008, 31, 1665.

16. Fraceto, L. F.; Rosa, A. H.; Paula, E.; Moraes, C. M.; Quim. Nova 2008, $31,2152$.

17. Rolim-Neto, P.J.; Lavra, Z. M. M.; Medeiros, F. P. M.; Silva, R. M. F.; Quim. Nova 2008, 31, 969 . 


\section{DESENVOLVIMENTO E VALIDAÇÃO DE MÉTODO ANALÍTICO POR CROMATOGRAFIA LÍQUIDA DE ALTA EFICIÊNCIA (CLAE) PARA DETERMINAÇÃO DE ASSOCIAÇÃO DE AMPICILINAS EM PÓ LIOFILIZADO PARA INJEÇÃO}

Ana Paola Prata Cione*, Márcio José Liberale e Paulo Marcos da Silva

Laboratório de Fármacos, Bioagri Laboratórios, Rod. Rio Claro-Piracicaba, SP 127, km 24, 13412-000 Piracicaba - SP, Brasil

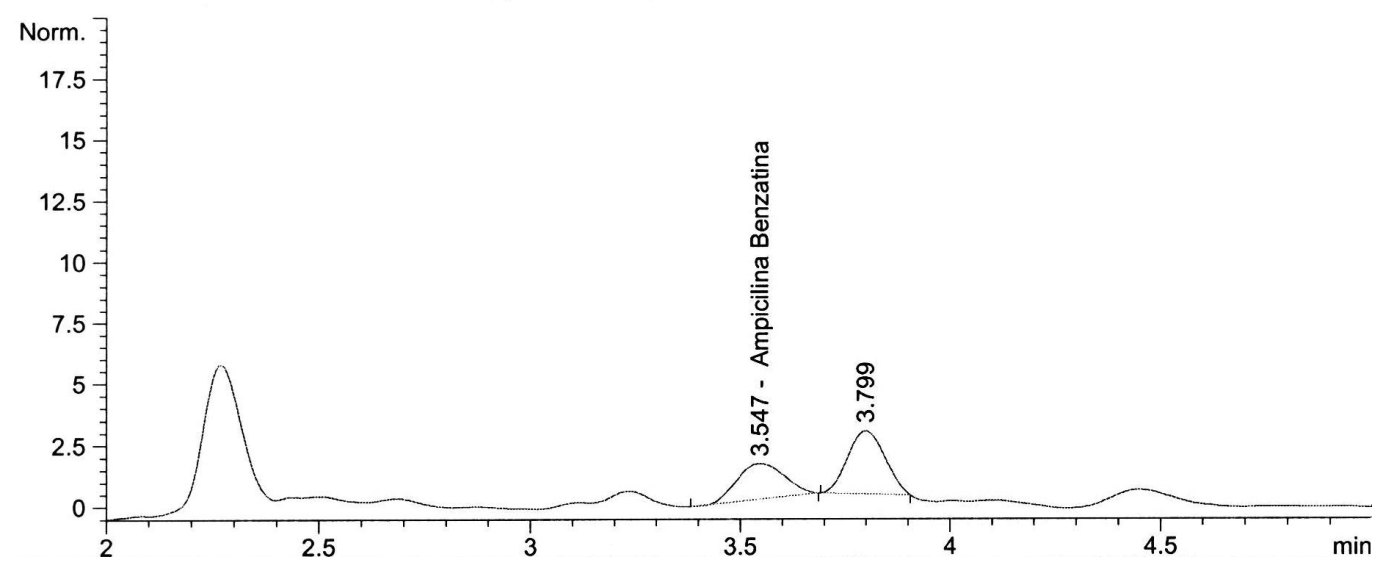

Figura 1S. Cromatograma representativo ensaio seletividade (degradação por oxidação por aquecimento em $60{ }^{\circ} \mathrm{C}$

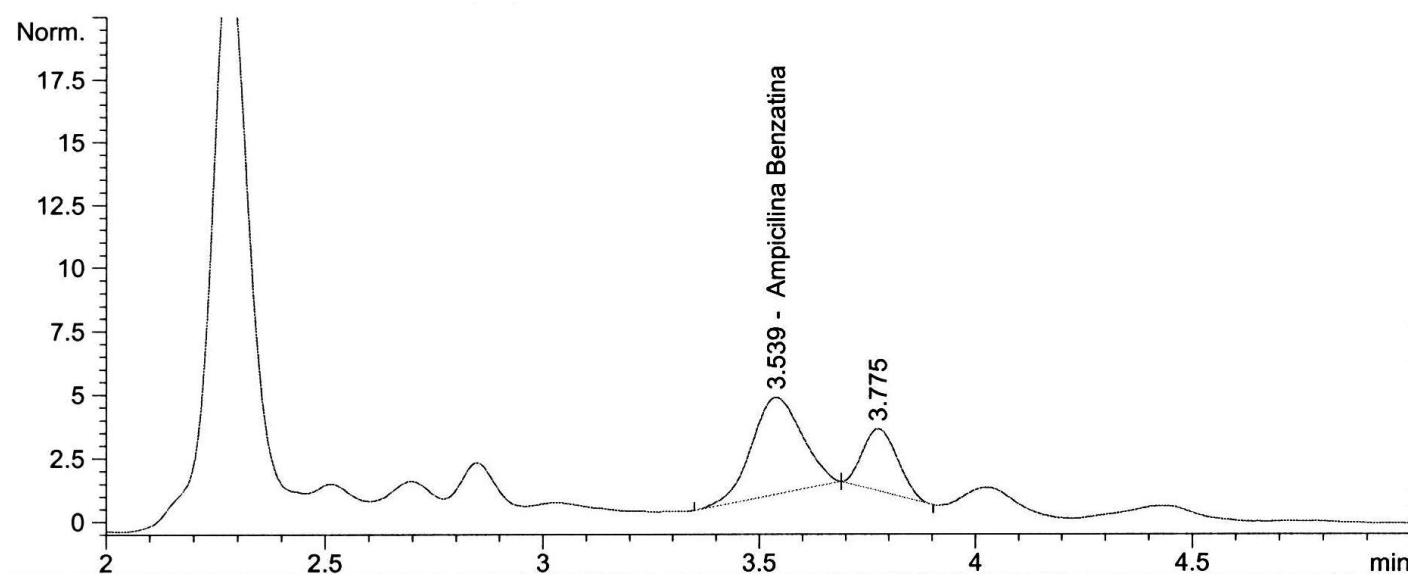

Figura 2S. Cromatograma representativo ensaio seletividade (degradação por oxidação por $\mathrm{H}_{2} \mathrm{O}_{2} \mathrm{O}, 3 \% \mathrm{v} / \mathrm{v}$ )

Tabela 1S. Resultados do ensaio de Robustez expressos em média \pm DPR\% em relação aos resultados médios da Precisão - Benzatina e Ampicilina total

\begin{tabular}{|c|c|c|c|c|}
\hline & 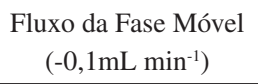 & $\begin{array}{l}\text { Fluxo da Fase Móvel } \\
\qquad\left(+0,1 \mathrm{~mL} \mathrm{~min}^{-1}\right)\end{array}$ & $\begin{array}{l}\text { Proporção da Fase Móvel } \\
\qquad(-5 \%)\end{array}$ & $\begin{array}{l}\text { Proporção da Fase Móvel } \\
\qquad(+5 \%)\end{array}$ \\
\hline \multicolumn{5}{|c|}{ Benzatina (mg/amp.) } \\
\hline Robustez: & $173,01 \pm 0,41$ & $173,77 \pm 0,48$ & $173,66 \pm 0,46$ & $173,71 \pm 0,25$ \\
\hline \multicolumn{5}{|c|}{ Ampicilina total (mg/amp.) } \\
\hline Robustez: & $221,76 \pm 0,30$ & $221,61 \pm 0,27$ & $221,33 \pm 0,19$ & $233,27 \pm 0,22$ \\
\hline
\end{tabular}

*e-mail: anapaola@bioagri.com.br 